\title{
Josephson traveling-wave parametric amplifier with three-wave mixing
}

\author{
A. B. Zorin \\ Physikalisch-Technische Bundesanstalt, Bundesallee 100, 38116 Braunschweig, Germany and \\ Department of Physics, Lomonosov Moscow State University, Moscow, 119991, Russia
}

(Dated: February 26, 2016, revised August 2, 2016)

\begin{abstract}
We develop a concept of the traveling-wave Josephson parametric amplifier exploiting quadratic nonlinearity of a serial array of one-junction SQUIDs embedded in a superconducting transmission line. The external magnetic flux applied to the SQUIDs makes it possible to efficiently control the shape of their current-phase relation and, hence, the balance between quadratic and cubic (Kerrlike) nonlinearities. This property allows us to operate in the favorable three-wave-mixing mode with minimal phase mismatch, an exponential dependence of the power gain on number of sections $N$, a large bandwidth, a high dynamic range, and substantially separated signal $\left(\omega_{s}\right)$ and pump $\left(\omega_{p}\right)$ frequencies obeying relation $\omega_{s}+\omega_{i}=\omega_{p}$, where $\omega_{i}$ is the idler frequency. An estimation of the amplifier characteristics with typical experimental parameters, a pump frequency of $12 \mathrm{GHz}$, and $N=300$ yields a flat gain of $20 \mathrm{~dB}$ in the bandwidth of $5.6 \mathrm{GHz}$.
\end{abstract}

PACS numbers: 84.30.Le, 85.25.Cp, 05.45.-a, 74.81.Fa

\section{INTRODUCTION}

These days, the Josephson parametric amplifiers (JPAs) 1] have practically achieved a quantum-limited performance [2 4] and are considered to be the most advanced tools available for fine experiments in the field of quantum measurements [5, 6] and quantum-information technologies [7-10]. Recently, owing to impact of the kinetic-inductance traveling-wave parametric amplifier [1], the Josephson traveling-wave parametric amplifiers (JTWPAs) enabling larger gain per unit length with lesser pump power have been in the particular focus of several research groups [12 16]. Moreover, these promising devices have already demonstrated the performance with noise approaching the quantum limit (see the works of White et al. 14 and Macklin et al. 16 ). In contrast to conventional JPAs including Josephson junctions (JJs) embedded in superconducting cavities and, therefore, suffering from inherent gain-bandwidth trade-off, JTWPAs are designed as microwave transmission lines enabling the mixing of propagating microwaves and, therefore, free of the bandwidth limitation and allowing higher dynamic range. These properties are required particularly for the frequency-multiplexing readout of quantum objects [17].

Similar to the concept of parametric amplification in nonlinear optical fibers 18], JTWPAs analyzed [12, 13, 15] and accomplished [14, 16] to date were based on the Kerr nonlinearity, i.e. on the dependence of the refractive index $n$ on intensity of the wave $\propto|\boldsymbol{E}|^{2}$. In superconducting circuits, this nonlinearity is due to the dependence of the Josephson inductance (equivalent to refractive index $n$ in optics) on the square of current $I^{2}$, viz. $L_{J}(I) \approx \Phi_{0}\left(1+\tilde{\gamma} I^{2} / I_{c}^{2}\right) /\left(2 \pi I_{c}\right)$, where $\Phi_{0}$ is the magnetic flux quantum and $I_{c}$ is the Josephson critical current. The nonlinear term originates from the term $\propto \varphi^{3}$ in the Taylor expansion of the Josephson supercurrent $I_{J}=I_{c} \sin \varphi$. Generally, by exploiting the centrosymmetric nonlinearity of the supercurrent, $I_{J}(-\varphi)=-I_{J}(\varphi)$ or, equivalently, the symmetric nonlinearity of inductance, $L_{J}(-I)=L_{J}(I)$, JTWPAs can, however, only operate in the four-wave-mixing mode [18]; i.e., when the signal $\left(\omega_{s}\right)$, idler $\left(\omega_{i}\right)$ and pump $\left(\omega_{p}\right)$ tones obey the relation $\omega_{s}+\omega_{i}=2 \omega_{p}$.

In this paper, we propose a JTWPA possessing nonzero quadratic nonlinearity produced by nonlinear inductance $L_{J}(I)$, the power expansion of which contains a term proportional to $I / I_{c}$ (or index $n$ having dependence on $\boldsymbol{E}$ ). We engineer this nonlinearity by means of modification of a current-phase relation in a superconducting circuit including JJs. This property enables operation in a favorable, three-wave-mixing mode (whose theory had been developed by Cullen as early as 1960 [19]) with frequen-

(a)

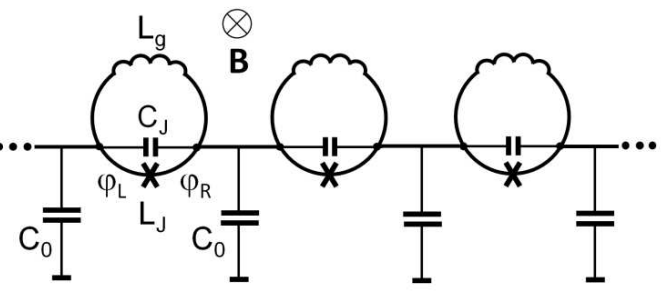

(b)

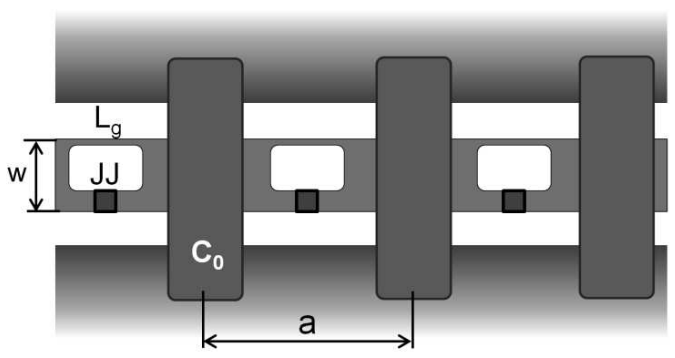

FIG. 1: (a) Electric diagram of the transmission line including an array of one-junction SQUIDs. The tunnel JJ is presented as the parallel connection of Josephson inductance $L_{J}$ and tunnel capacitance $C_{J}$. (b) Possible layout of the transmission line with parallel plate capacitor cross-overs. 
cies obeying the relation

$$
\omega_{s}+\omega_{i}=\omega_{p}
$$

For such a regime the pump frequency shifts away substantially from the signal band and therefore can be efficiently filtered out from the amplified signal. Because of inherently stronger quadratic interaction (in comparison to higher-order cubic interaction), efficient operation in the three-wave-mixing mode is possible with a smaller pump power. Moreover, the three-wave-mixing mode enables a high dynamic range and wide-band operation. These promising characteristics have been recently demonstrated in the experiment with a NbTiN kinetic-inductance traveling-wave amplifier [20] in which quadratic nonlinearity was created in addition to conventional Kerr nonlinearity of a thin superconducting wire by means of applying a dc current bias.

\section{THE MODEL}

The proposed ladder-type transmission line having full control of both quadratic and cubic nonlinearities consists of a serial array of one-junction SQUIDs, or the so-called rf-SQUIDs, embedded in the central conductor of the coplanar waveguide as shown in Fig. 1. The value of the screening SQUID parameter $\beta_{L} \equiv 2 \pi L_{g} I_{c} / \Phi_{0}<1$ [21], where $L_{g}$ is the geometrical inductance. In this case, the external magnetic flux $\Phi_{e}$ induces a flux inside the loop $\Phi_{\mathrm{dc}}\left(\Phi_{e}\right)$ which is a single-valued function of $\Phi_{e}$, and its value is found by solving the transcendental equation (see, for example, Ref. [22])

$$
\Phi_{\mathrm{dc}}+\left(\Phi_{0} / 2 \pi\right) \beta_{L} \sin \left(2 \pi \Phi_{\mathrm{dc}} / \Phi_{0}\right)=\Phi_{e} .
$$

The phase drop across the JJ (see Fig. 1a) is therefore $\varphi_{\mathrm{dc}} \equiv \varphi_{\mathrm{L}}-\varphi_{\mathrm{R}}=2 \pi \Phi_{\mathrm{dc}} / \Phi_{0}$. The inverse (linear) inductance for a small input current is $L^{-1}=L_{g}^{-1}+L_{J}^{-1}=$ $L_{g}^{-1}\left(1+\beta_{L} \cos \varphi_{\mathrm{dc}}\right)$, whereas the current-phase relation for the flux-biased SQUID is expressed by the formula

$$
I(\varphi)=I_{c} \varphi / \beta_{L}+I_{c}\left[\sin \left(\varphi_{\mathrm{dc}}+\varphi\right)-\sin \varphi_{\mathrm{dc}}\right] .
$$

Here, $\varphi$ is the variation of the JJ phase associated with the (ac) current injected in the SQUID. By expending the current $I(\varphi)$ in a power series of the small parameter $|\varphi| \ll 1$, one arrives at the formula

$$
I / I_{c}=\left(\beta_{L}^{-1}+\cos \varphi_{\mathrm{dc}}\right) \varphi-\tilde{\beta} \varphi^{2}-\tilde{\gamma} \varphi^{3}-\ldots,
$$

with $\tilde{\beta}=\frac{1}{2} \sin \varphi_{\mathrm{dc}}, \tilde{\gamma}=\frac{1}{6} \cos \varphi_{\mathrm{dc}}$, etc. The first term on the right-hand side is related to the inverse linear inductance of the SQUID, whereas $\tilde{\beta}$ and $\tilde{\gamma}$ describe the quadratic and Kerr (cubic) nonlinearities, respectively. The quadratic nonlinearity introduces desired asymmetry $I(-\varphi) \neq-I(\varphi)$ and allows a number of remarkable physical effects inaccessible with only Kerr nonlinearity.
These include, for example, the second harmonic generation (SHG) 23], spontaneous parametric down conversion (SPDC) [24 26], and what we focus on here, the three-wave mixing that enables parametric gain [19].

Following the method of deriving a wave equation for a ladder-type $L C$ transmission line with embedded JJs, which was described in detail by Yaakobi et al. [12], we arrive in our case at the equation for the phase $\phi(x, t)$ on the circuit nodes,

$$
\begin{aligned}
& \frac{\partial^{2} \phi}{\partial x^{2}}- \omega_{0}^{-2} \frac{\partial^{2} \phi}{\partial t^{2}}+\omega_{J}^{-2} \frac{\partial^{4} \phi}{\partial x^{2} \partial t^{2}} \\
&+\beta \frac{\partial}{\partial x}\left[\left(\frac{\partial \phi}{\partial x}\right)^{2}\right]+\gamma \frac{\partial}{\partial x}\left[\left(\frac{\partial \phi}{\partial x}\right)^{3}\right]=0 .
\end{aligned}
$$

Here $x=X / a$ is a dimensionless coordinate; $a$, the section size; $\omega_{0}=\left(L C_{0}\right)^{-\frac{1}{2}}$, the cutoff frequency; and, $\omega_{J}=\left(L C_{J}\right)^{-\frac{1}{2}}$, the plasma frequency of the SQUID. The ac part of the phase on a SQUID is $\varphi=a \frac{\partial \phi}{\partial X}=\frac{\partial \phi}{\partial x}$, whereas voltage on a ground capacitor is $V=\left(\Phi_{0} / 2 \pi\right) \frac{\partial \phi}{\partial t}$. The nonlinear coefficients are $\beta=\tilde{\beta} \beta_{L}$ and $\gamma=\tilde{\gamma} \beta_{L}$ (see their plots in Fig. 2).

The third term in Eq. (15) yields an ordinary superlinear frequency dependence [12, [15] of the dimensionless wave vector $k=2 \pi a / \lambda$,

$$
k(\omega)=\frac{\omega}{\omega_{0} \sqrt{1-\omega^{2} / \omega_{J}^{2}}},
$$

which follows from a plane wave solution $e^{i(k x-\omega t)}$ of the corresponding linear equation (at $\beta=\gamma=0$ ). The

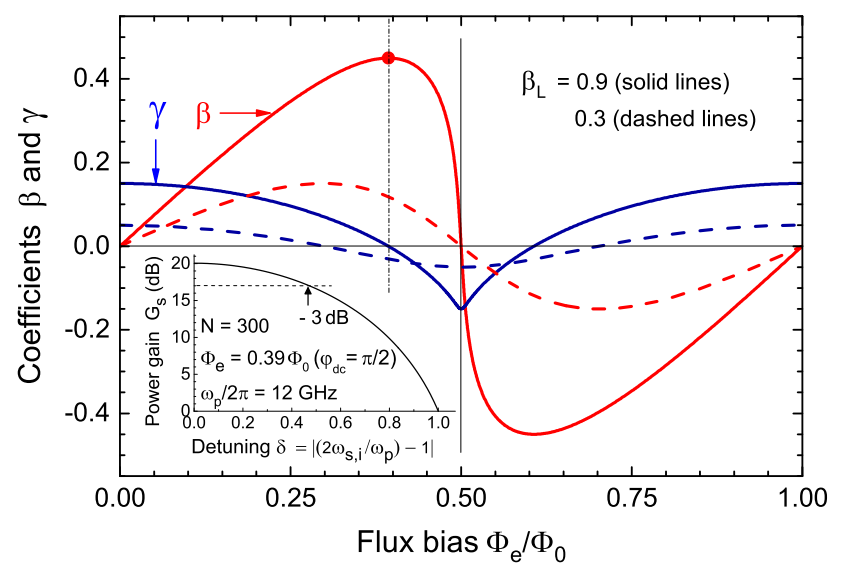

FIG. 2: Coefficients $\beta$ (red curves) and $\gamma$ (blue curves) as functions of applied magnetic flux $\Phi_{e}$ for two values of SQUID parameter $\beta_{L}$. The thin dotted vertical line indicates optimal flux bias $\Phi_{e}=0.39 \Phi_{0}$ ensuring the phase $\varphi_{\mathrm{dc}}=\pi / 2$ corresponding to maximum gain (maximum value of $|\beta|$ and zero value of $\gamma$ ) for the case of $\beta_{L}=0.9$. Inset shows the frequency dependence of signal-power gain $G_{s}$ in the JTWPA, calculated for typical experimental parameters and the optimum flux bias. 
section size $a$ is assumed to be much smaller than the wavelengths of propagating signals, i.e. $a \ll \lambda$, the values of $k \ll 1$ and, therefore, all working frequencies are small, $\omega_{s, i, p} \ll \omega_{0}$. For sufficiently large plasma frequency $\omega_{J} \gg \omega_{s, i, p}$, the chromatic dispersion Eq. (6) is small, $k(\omega) \approx\left(\omega / \omega_{0}\right)\left(1+0.5 \omega^{2} / \omega_{J}^{2}\right)$, and, as usual in optics [18], positive, $\operatorname{dn}(\omega) / d \omega>0$.

\section{ANALYSIS AND CHARACTERISTICS}

\section{A. Signal gain}

The solution of the wave equation (5) is found using the coupled-mode-equation (CME) method [18] in the form

$$
\phi(x, t)=\frac{1}{2} \sum_{j=\{s, i, p\}}\left[A_{j}(x) e^{i\left(k_{j} x-\omega_{j} t\right)}+\text { c.c. }\right],
$$

where $k_{s, i, p}$ are wave vectors and $A_{s, i, p}(x)$ are slowly varying (i.e. $\left|\frac{\partial^{2} A_{j}}{\partial x^{2}}\right| \ll k_{j}\left|\frac{\partial A_{j}}{\partial x}\right| \ll k_{j}^{2}\left|A_{j}\right|, j=\{s, i, p\}$ ) amplitudes of the signal, idler and pump waves, respectively. Corresponding CMEs take the form

$$
\begin{aligned}
\frac{d A_{p}}{d x} & =i \frac{3}{8} \gamma k_{p}^{3} A_{p}\left|A_{p}\right|^{2}-\frac{\beta}{2} k_{s} k_{i} A_{s} A_{i} e^{-i \Delta k x} \\
\frac{d A_{s, i}}{d x} & =i \frac{3}{4} \gamma k_{s, i} k_{p}^{2} A_{s, i}\left|A_{p}\right|^{2}+\frac{\beta}{2} k_{i, s} k_{p} A_{i, s}^{*} A_{p} e^{i \Delta k x}
\end{aligned}
$$

The phase mismatch resulted from dispersion equation (6) is

$$
\Delta k=k_{p}-k_{s}-k_{i} \approx \frac{\omega_{p}^{3}-\omega_{s}^{3}-\omega_{i}^{3}}{2 \omega_{0} \omega_{J}^{2}}=\frac{3 \omega_{s} \omega_{i} \omega_{p}}{2 \omega_{0} \omega_{J}^{2}}>0,
$$

where the higher-order small terms $\propto \omega_{0}^{-1} \omega_{J}^{-4} \omega_{s, i, p}^{5}$ are neglected. The terms proportional to $\gamma$ produce the selfphase modulation (SPM) $\vartheta_{p}$ and the cross-phase modulations $(\mathrm{XPM}) \vartheta_{s}$ and $\vartheta_{i}$, respectively [18], i.e.,

$$
\begin{aligned}
\vartheta_{p} & =(3 / 8) \gamma k_{p}^{2}\left|A_{p}\right|^{2} k_{p}, \\
\vartheta_{s, i} & =(3 / 4) \gamma k_{p}^{2}\left|A_{p}\right|^{2} k_{s, i},
\end{aligned}
$$

which contribute to the total phase mismatch

$$
\psi=\Delta k+\vartheta
$$

where

$$
\vartheta=\vartheta_{p}-\vartheta_{s}-\vartheta_{i} \approx-(3 / 8)\left(\omega_{p} / \omega_{0}\right)^{3} \gamma\left|A_{p}\right|^{2} .
$$

Here we had again neglected the higher-order terms $\propto$ $\left(\omega_{s, i, p} / \omega_{J}\right)^{2}\left(\omega_{p} / \omega_{0}\right)^{3} \gamma$ because of a small $\gamma$ and relations $k_{s, i, p} \approx \omega_{s, i, p} / \omega_{0}$.

Under the undepleted-pump assumption, $\left|A_{p}(x)\right|=$ $A_{p 0} \gg\left|A_{s, i}(x)\right|$ the pump can be found from Eq. (8) explicitly,

$$
A_{p}(x)=A_{p 0} e^{i \chi_{p 0}} e^{i \vartheta_{p} x}
$$

where $\chi_{p 0}$ is the initial phase. Then Eq. (9) is simplified:

$$
\begin{aligned}
\frac{d A_{s, i}}{d x} & =i \vartheta_{s, i} A_{s, i}+2 g_{0} \frac{\omega_{i, s}}{\omega_{p}} A_{i, s}^{*} e^{i \chi_{p 0}} e^{i \vartheta_{p} x} e^{i \Delta k x}, \\
g_{0} & =|\beta| A_{p 0} \omega_{p}^{2} / 4 \omega_{0}^{2} .
\end{aligned}
$$

For the zero initial idler, $A_{i}(0)=0$, but nonzero initial signal, $A_{s}(0)=A_{s 0} e^{i \chi_{s 0}} \neq 0$, the solution of linear equations (16) can be presented in the form [18]

$$
\begin{aligned}
& A_{s}(x)=A_{s 0} e^{i \chi_{s 0}}\left[\cosh (g x)-\frac{i \psi}{2 g} \sinh (g x)\right] e^{i\left(\vartheta_{s}+\frac{\psi}{2}\right) x} \\
& A_{i}(x)=2 \frac{g_{0} \omega_{s}}{g \omega_{p}} A_{s 0} e^{i \chi_{i 0}} e^{-i \chi_{p 0}} \sinh (g x) e^{i\left(\vartheta_{i}+\frac{\psi}{2}\right) x}
\end{aligned}
$$

with initial phases obeying the relation $\chi_{p 0}-\chi_{s 0}-\chi_{i 0}=$ 0 . The complex exponential gain factor is

$$
g=\left[\left(1-\delta^{2}\right) g_{0}^{2}-(\psi / 2)^{2}\right]^{\frac{1}{2}},
$$

where $1-\delta^{2}$ stands for $4 \omega_{s} \omega_{i} / \omega_{p}^{2}$. Here the dimensionless detuning is

$$
\delta=\left|2 \omega_{s, i}-\omega_{p}\right| / \omega_{p},
$$

while the total phase mismatch equals

$$
\psi=\Delta k+\vartheta=\frac{3 \omega_{p}^{3}}{8 \omega_{0}^{3}}\left[\left(1-\delta^{2}\right) \frac{\omega_{0}^{2}}{\omega_{J}^{2}}-\gamma A_{p 0}^{2}\right] .
$$

For the zero phase mismatch $\psi=0$, the signal has the maximum power gain

$$
G_{s}=\left|A_{s} / A_{s 0}\right|^{2}=1+\sinh ^{2}\left(\sqrt{1-\delta^{2}} g_{0} N\right)
$$

where $N$ is the length of the line.

\section{B. Phase mismatch}

Generally, realization of zero phase mismatch is a challenging problem in designing four-wave-mixing JTWPAs. The main difficulty is that both the phase modulation and the gain depend on the very same parameter, i.e. the Kerr nonlinearity $\gamma$. This problem can be partially solved by very neat engineering of dispersion using auxiliary resonance elements embedded in the transmission line [13, 14]. In contrast to this situation, in our threewave-mixing case the phase-modulation effects and signal gain are controlled by two different nonlinear terms, i.e. $\propto \gamma A_{p 0}^{2}$ and $\propto \beta A_{p 0}$, respectively, so a phase matching can be realized by simply adjusting external flux bias $\Phi_{e}$ (see the dependencies $\beta\left(\Phi_{e}\right)$ and $\gamma\left(\Phi_{e}\right)$ in Fig. 2).

In particular, for $\omega_{s} \approx \omega_{i} \approx 0.5 \omega_{p}$ (i.e. small $\delta \approx 0$ ) the total phase mismatch $\psi$ Eq. (22) can be made equal to zero if, first, $\gamma>0$ and, second, $\gamma A_{p 0}^{2}$ is of the order of $\omega_{0}^{2} / \omega_{J}^{2}=C_{J} / C_{0}$. Assuming that the optimal pump amplitude is determined by a swing of the Josephson phase $\varphi_{a} \sim 1$, i.e. $A_{p 0}=\varphi_{a} / k_{p} \approx \omega_{0} / \omega_{p}$, we 
obtain $\gamma \approx \omega_{p}^{2} / \omega_{J}^{2} \ll 1$. In this case, finite detuning $\delta$ may cause phase mismatch $|\psi| \approx(3 / 8) \delta^{2} \omega_{p}^{3} /\left(\omega_{0} \omega_{J}^{2}\right)$ which is much smaller than the exponential gain factor $g=0.5 \sqrt{1-\delta^{2}}|\beta| \omega_{p} / \omega_{0}$ (of course, not for vanishingly small values of $|\beta|$ and a value $\delta$ not very close to 1 ). Thus, for proper circuit parameters, an exponential gain with frequency dependence given by Eq. (23) is always possible.

Large exponential power gain allows operating even in the regime of imperfect phase matching, $\psi \neq 0$. The dephasing length on which the phase mismatch becomes substantial is

$$
N_{\psi}=\frac{\pi}{|\psi|}=\frac{8 \pi \omega_{0}^{3}}{3 \omega_{p}^{3}}\left|\frac{\left(1-\delta^{2}\right) \omega_{0}^{2}}{\omega_{J}^{2}}-\gamma A_{p 0}^{2}\right|^{-1} .
$$

This formula can be rewritten as

$$
N_{\psi}=\frac{N_{\mathrm{D}} N_{\mathrm{PM}}}{\left|N_{\mathrm{D}}-N_{\mathrm{PM}}\right|}=\left|N_{\mathrm{D}}^{-1}-N_{\mathrm{PM}}^{-1}\right|^{-1},
$$

where

$$
N_{\mathrm{D}}=\frac{8 \pi \omega_{0} \omega_{J}^{2}}{3 \omega_{p}^{3}\left(1-\delta^{2}\right)}
$$

and

$$
N_{\mathrm{PM}}=\frac{8 \pi \omega_{0}^{3}}{3 \gamma \omega_{p}^{3} A_{p 0}^{2}}
$$

are the dephasing lengths attributed solely to chromatic dispersion and attributed solely to SPM and XPM, respectively. For optimal values of $|\beta|=\beta_{L} / 2$ and $\gamma \rightarrow 0$ (see Fig. 2) the length $N$ which is sufficient for attaining designed value of gain $G_{s}$,

$$
N=\frac{\operatorname{arccosh} \sqrt{G_{s}}}{g} \approx \frac{4 \omega_{0}^{2} \ln \left(2 \sqrt{G_{s}}\right)}{|\beta| \omega_{p}^{2}\left(1-\delta^{2}\right) A_{p 0}},
$$

can safely be designed substantially smaller than very large $N_{\psi}$. In this case the gain suppression because of the pump depletion may dominate.

\section{Pump depletion}

Depletion of the pump power in this JTWPA may occur by means of two major mechanisms. Firstly, there is a possible leak of power to higher harmonics, especially to the second harmonic $2 \omega_{p}$. An analysis of the corresponding CME shows that because of weak frequency dispersion $\left(2 \omega_{p} \ll \omega_{J}\right)$ and both small SPM and XPM $(\gamma \ll 1)$ the phase mismatch between the main tone and the second harmonics is small. Without special precautions this fact may cause intensive SHG. To suppress SHG one may create a narrow stopband around $2 \omega_{p}$ by applying, for example, the technique of periodic variation of wave impedance developed by Eom et al. 11]. In our case, such stopband engineering is reduced to some change of, for example, capacitance $C_{0}$ in every $m$-th section of the line. The number $m$ should correspond to a half wavelength of a preselected frequency, i.e., $m=\left[2 \pi \omega_{0} / 4 \omega_{p}\right]$. Alternatively, one can reduce the cutoff frequency $\omega_{0}$ to prevent propagation of higher $(\geq 2)$ pump harmonics having frequencies larger than $\omega_{0}$, as was done in Ref. [14].

Secondly, the input pump power $P_{p 0}$ is inevitably converted into the power of signal $P_{s}$ and idler $P_{i}\left(P_{j} \propto\right.$ $\left.\left(\frac{\partial \phi_{j}}{\partial t}\right)^{2}=\omega_{j}^{2}\left|A_{j}\right|^{2}, j=\{s, i, p\}\right)$, i.e.

$$
\omega_{p}^{2} A_{p 0}^{2}+\omega_{s}^{2} A_{s 0}^{2}=\omega_{p}^{2}\left|A_{p}\right|^{2}+\omega_{s}^{2}\left|A_{s}\right|^{2}+\omega_{i}^{2}\left|A_{i}\right|^{2} .
$$

Specifically, for a zero phase mismatch, the power gain versus the length dependence is described by the formula (see the Appendix for details)

$$
G_{s}^{(\mathrm{d})}=\frac{1}{\operatorname{dn}^{2}(g N / \mathrm{k}, \mathrm{k})} \rightarrow 1+\sinh ^{2}(g N), \text { as } \mathrm{k} \rightarrow 1,
$$

where $\operatorname{dn}(u, \mathrm{k})$ is the Jacobi elliptic function with modulus value

$$
\mathrm{k}=\sqrt{\omega_{p} A_{p 0}^{2} /\left(\omega_{s} A_{s 0}^{2}+\omega_{p} A_{p 0}^{2}\right)} .
$$

The maximum possible power gain $G_{s}^{\max }=1 /\left(1-\mathrm{k}^{2}\right)$ is achieved for the line length $N=\mathrm{k} K(\mathrm{k}) / g$, where $K$ is the complete elliptic integral of the first kind. For a longer line the gain is reduced due to reverse power conversion from the signal and the idler to the pump [19].

\section{POSSIBLE DESIGN AND DISCUSSION}

Finally, let us make an estimation of possible experimental parameters. For state-of-the-art four-wavemixing counterparts critical current $I_{c}=5 \mu \mathrm{A}$ [14, 16]. Assuming a similar value of $I_{c}$ and a critical-current density of $500 \mathrm{~A} / \mathrm{cm}^{2}$, the tunnel area is about $1 \mu \mathrm{m}^{2}$ and JJ capacitance $C_{J} \approx 60 \mathrm{fF}$. For a width of center strip of $w=15 \mu \mathrm{m}$ capacitance $C_{0}$ can be designed [4] to be around $100 \mathrm{fF}$ without significant increase of the section size $a \sim 2 w$. By applying small meandering geometrical inductance $L_{g}$ can be made to be about $57 \mathrm{pH}$, yielding $\beta_{L} \approx 0.9$. For appropriate flux bias corresponding to $\varphi_{\mathrm{dc}}=\pi / 2$ and ensuring maximum value of $\beta=\beta_{L} / 2=0.45$ and zero $\gamma$ (see Eq. (2)), the resulting inductance $L=L_{g} /\left(1+\cos \varphi_{\mathrm{dc}}\right)=L_{g}=57 \mathrm{pH}$.

The wave impedance of this line is, therefore, $Z_{0}=$ $\left(L / C_{0}\right)^{\frac{1}{2}} \approx 24 \Omega$, plasma frequency $\omega_{J} / 2 \pi \approx 86 \mathrm{GHz}$ and the cutoff frequency $\omega_{0} / 2 \pi \approx 67 \mathrm{GHz}$. Taking the pump frequency $\omega_{p} / 2 \pi=12 \mathrm{GHz}$ and amplitude $A_{p 0}=0.5 \omega_{0} / \omega_{p} \approx 2.8$, corresponding to phase swing $\varphi_{a}=k_{p} A_{p 0}=0.5 \mathrm{rad} \lesssim 30^{\circ}$ and current $I_{p 0}^{\mathrm{rms}}=$ $\frac{1}{\sqrt{2}}\left(\Phi_{0} / 2 \pi L\right) \varphi_{a} \approx 1.97 \mu \mathrm{A}(\approx-70.3 \mathrm{dBm})$, we obtain the factor $g_{0}=|\beta| \omega_{p} / 8 \omega_{0} \approx 0.01$ (see Eq. (17)). The power gain of $N$-section JTWPA given by Eq. (23) is, therefore, $G_{s} \approx 1+\sinh ^{2}(0.01 N)$, i.e. about $20 \mathrm{~dB}$ for $N=300$, 


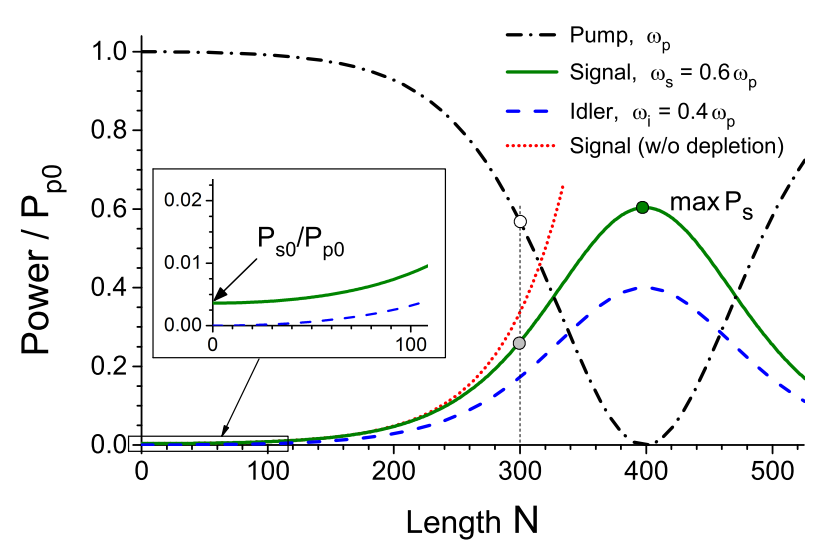

FIG. 3: Amplification of signal (green solid curve) and idler (dashed blue curve), and depletion of pump (black dashdotted curve) versus the line length $N$ found by numeric solving of CMEs. (A1)-(A3) in the case of $\omega_{s}=0.6 \omega_{p}$. The gray and open circles show the output powers of the signal and the pump, respectively, corresponding to a $1 \mathrm{~dB}$ suppression of the nominal gain of $20 \mathrm{~dB}$ in the 300-section array occurred at $\mathrm{k}^{\prime}=0.075$. For the pump power $P_{p 0}=-70.3 \mathrm{dBm}$ the signal-saturation power is $P_{s 0}=\left(\mathrm{k}^{\prime} / \mathrm{k}\right)^{2}\left(\omega_{s} / \omega_{p}\right) P_{p 0} \approx$ $0.0057\left(\omega_{s} / \omega_{p}\right) P_{p 0} \approx-96 \mathrm{dBm}$. For comparison, red dotted curve shows the exponential rise (described by Eq. (23)) of this signal in the case of undepleted pump. The solid green circle shows maximum signal power $P_{s}=\left(\omega_{s} / \omega_{p}\right) P_{p 0} \approx$ $-73.5 \mathrm{dBm}$ corresponding to a maximum achievable gain of about $22.5 \mathrm{~dB}\left(\right.$ at $\left.N=N_{m}=399\right)$.

whereas the geometrical length $a N \approx 9 \mathrm{~mm}$. This length corresponds to $n_{\lambda}=a N / \lambda_{p}=N /\left(2 \pi \omega_{0} / \omega_{p}\right) \approx 8.6$ wavelengths of the pump.

The evaluated dephasing lengths in Eqs.(26) and (27) are $N_{\mathrm{D}}=2400$ and $N_{\mathrm{PM}} 186 / \gamma$, respectively. Taking the most unfavorable value of $\gamma=-0.1$ (achieved instantly only at the extreme position of an oscillating phase $\varphi$ ), we have a very conservative estimate Eq.(25) for a total dephasing length $N_{\psi} \approx 1000 \gg N$. The dependence of the power gain $G_{s}$ on frequency (shown in the inset of Fig. 2) yields a remarkably wide $3 \mathrm{~dB}$ range of $0.47 \omega_{p} / 2 \pi \approx$ $5.64 \mathrm{GHz}$. While this $3 \mathrm{~dB}$ range is only slightly larger than that achieved in the four-wave mixing JTWPA (e.g., $\approx 0.42 \omega_{p} / \pi$ [15], $0.38 \omega_{p} / \pi$ [14] and $0.21 \omega_{p} / \pi$ [16]), the latter typically exhibits considerable ripple or other variations in the gain versus frequency, which can be practically cumbersome. Moreover, the contiguous frequency range of the power gain in our JTWPA is extended up to a pump frequency of $\omega_{p} / 2 \pi=12 \mathrm{GHz}$.

As follows from the corresponding coupled equations (A1)-(A3) (see the Appendix) the $1 \mathrm{~dB}$ reduction of the gain caused by pump depletion occurs when the complementary modulus value $\mathrm{k}^{\prime} \equiv \sqrt{1-\mathrm{k}^{2}} \approx 0.075$. The simulated dependence of the signal and pump powers versus the length $N$ presented in Fig. 3 shows that, for a rather modest pump power of $-70.3 \mathrm{dBm}$, the input signalsaturation power is $P_{s 0} \approx-96 \mathrm{dBm}$ (cf. $-98 \mathrm{dBm}$ for a
20-dB gain in Refs. [13, 15] and $-92 \mathrm{dBm}$ for a $12-\mathrm{dB}$ gain in Ref. [14]). The dynamic range can be further improved by an increase of the phase swing $\varphi_{a}$ from $0.5 \mathrm{rad}$ up to, for example, $1 \mathrm{rad}$ with a corresponding 6 - $\mathrm{dB}$ increase of both the pump and the signal-saturation powers, i.e., up to approximately $-64.3 \mathrm{dBm}$ and $-90 \mathrm{dBm}$, respectively.

For the above-mentioned experimental parameters, the line impedance $Z_{0}$, is still below $50 \Omega$ which may require special matching with a signal source and a postamplifier. This matching can be done, for example, with the help of a Klopfenstein taper [27] having a design similat to that in Ref. [4]. The low- $Z_{0}$ problem can be solved (at a price of some reduction of dynamic range and larger size of the circuit) by simultaneously making ground capacitance $C_{0}$ and the critical current $I_{c}$ smaller and the geometrical inductance $L_{g}$ larger. For example, $I_{c}=2.5 \mu \mathrm{A}, L_{g}=114 \mathrm{pH}$ (which can be realized by means of a relatively large size inductor) and $C_{0}=50 \mathrm{fF}$ should yield $Z_{0} \approx 50 \Omega$, while keeping $\beta_{L}, \omega_{J}$ and $\omega_{0}$ equal to the above-mentioned design values. To keep the length of such circuit reasonably short one can, for example, replace large geometrical inductance $L_{g} \propto 1 / I_{c}$ by kinetic inductance of a serial array of two to four larger JJs. Finally, another strategy for increasing $Z_{0}$ toward $50 \Omega$ is replacing a single rf-SQUID in each section by a group of two to four serially connected rf-SQUIDs.

\section{CONCLUSION}

We have developed a concept of a JTWPA with three-wave mixing, which can outperform state-of-the-art JTWPAs operating on the principle of four-wave mixing. Our circuit enables high gain, the widest flat bandwidth and nominally zero phase matching. Moreover, this JTWPA allows efficient operation with slightly imperfect phase matching. The proposed design is simple, compact, excludes the engineering of sophisticated resonant phase-matching elements, allows cascading and multiplexing, and is feasible in the labs with standard fabrication facilities.

This JTWPA flexibly allows further optimization of its parameters and possible integration with, for example, SQUID transducers, single-photon detectors, qubits, etc. We believe that this amplifier with potentially quantumlimited noise performance will advance high-fidelity measurements and signal processing at the single-photon level. Last, but not least, realization of a SPDC regime [26] in a circuit with a Josephson noncentrosymmetric nonlinear medium, like in this JTWPA, may allow its application for the generation of entangled photon pairs with frequencies obeying the relation $\omega_{1}+\omega_{2}=\omega_{p}$. Such entangled biphotons are of particular importance for creating a quantum processor, quantum-key distribution, and a secure transmission of data. 


\section{ACKNOWLEDGMENTS}

The author thanks R. Dolata, M. Khabipov, G. Kh. Kitaeva, M. Chekhova, P. Meeson, K. Porsch, P. Delsing, and J. Martinis for stimulating discussions. This work belongs partly to the Joint Research Project MICROPHOTON of the European Metrology Research Programme (EMRP). The EMRP is jointly funded by the EMRP participating countries within EURAMET and the European Union.

\section{APPENDIX: EFFECT OF PUMP DEPLETION}

Neglecting the SPM and XPM effects (i.e. $\gamma \rightarrow 0$ ) and the phase mismatch due to chromatic dispersion $(\Delta k \rightarrow 0)$ the coupled-mode equations (8) and (9) for the amplitudes and the phases of the waves, $A_{p, s, i}(x)=$ $\left|A_{p, s, i}(x)\right| e^{i \chi_{p, s, i}(x)}$, take the form:

$$
\begin{aligned}
\frac{d\left|A_{p}\right|}{d x} & =-\frac{\beta}{2} \frac{\omega_{s} \omega_{i}}{\omega_{0}^{2}}\left|A_{s}\right|\left|A_{i}\right| \cos \chi \\
\frac{d\left|A_{s}\right|}{d x} & =\frac{\beta}{2} \frac{\omega_{i} \omega_{p}}{\omega_{0}^{2}}\left|A_{i}\right|\left|A_{p}\right| \cos \chi \\
\frac{d\left|A_{i}\right|}{d x} & =\frac{\beta}{2} \frac{\omega_{s} \omega_{p}}{\omega_{0}^{2}}\left|A_{s}\right|\left|A_{p}\right| \cos \chi \\
\frac{d \chi}{d x} & =\frac{\beta}{2} \frac{\omega_{p} \omega_{s} \omega_{i}}{\omega_{0}^{2}} \\
& \times\left(\frac{\left|A_{s}\right|\left|A_{i}\right|}{\omega_{p}\left|A_{p}\right|}-\frac{\left|A_{p}\right|\left|A_{i}\right|}{\omega_{s}\left|A_{s}\right|}-\frac{\left|A_{s}\right|\left|A_{p}\right|}{\omega_{i}\left|A_{i}\right|}\right) \sin \chi
\end{aligned}
$$

where $\chi=\chi_{p}-\chi_{s}-\chi_{i}$. The initial conditions read $\left|A_{p}(0)\right|=A_{p 0},\left|A_{s}(0)\right|=A_{s 0},\left|A_{i}(0)\right|=0$ and $\chi(0)=$ $\chi_{p 0}-\chi_{s 0}-\chi_{i 0}=0$. Therefore, the fourth equation (A4) yields constant phase $\chi=0$, so $\cos \chi$ is equal to 1 . The set of equations (A1)-(A3) implies that

$$
-\omega_{p}\left|A_{p}\right| \frac{d\left|A_{p}\right|}{d x}=\omega_{s}\left|A_{s}\right| \frac{d\left|A_{s}\right|}{d x}=\omega_{i}\left|A_{i}\right| \frac{d\left|A_{i}\right|}{d x}
$$

or, equivalently, in terms of wave powers $\left(P_{j} \propto \omega_{j}^{2}\left|A_{j}\right|^{2}\right.$, $j=\{p, s, i\})$,

$$
-\frac{1}{\omega_{p}} \frac{d P_{p}}{d x}=\frac{1}{\omega_{s}} \frac{d P_{s}}{d x}=\frac{1}{\omega_{i}} \frac{d P_{i}}{d x} .
$$

Virtually, these are the Manley-Rowe relations for the waves [28]. The three conserved quantities (in the sense that they are spatially invariant) are

$$
\frac{P_{p}}{\omega_{p}}+\frac{P_{s}}{\omega_{s}}=M_{1}, \quad \frac{P_{p}}{\omega_{p}}+\frac{P_{i}}{\omega_{i}}=M_{2}, \quad \frac{P_{s}}{\omega_{s}}-\frac{P_{i}}{\omega_{i}}=M_{3} .
$$

Using the initial conditions, the constants $M_{1}, M_{2}$, and $M_{3}$ entering Eq. (A7) can be found, so the corresponding relations between the wave amplitudes read

$$
\begin{aligned}
\omega_{p}\left|A_{p}\right|^{2}+\omega_{s}\left|A_{s}\right|^{2} & =\omega_{p}\left|A_{p 0}\right|^{2}+\omega_{s}\left|A_{s 0}\right|^{2}, \\
\omega_{p}\left|A_{p}\right|^{2}+\omega_{i}\left|A_{i}\right|^{2} & =\omega_{p}\left|A_{p 0}\right|^{2}, \\
\omega_{s}\left|A_{s}\right|^{2}-\omega_{i}\left|A_{i}\right|^{2} & =\omega_{s}\left|A_{s 0}\right|^{2} .
\end{aligned}
$$

Substituting expressions

$$
\left|A_{s}\right|=\sqrt{\left|A_{s 0}\right|^{2}+\left(\omega_{p} / \omega_{s}\right)\left(\left|A_{p 0}\right|^{2}-\left|A_{p}\right|^{2}\right)}
$$

and

$$
\left|A_{i}\right|=\sqrt{\left(\omega_{p} / \omega_{i}\right)\left(\left|A_{p 0}\right|^{2}-\left|A_{p}\right|^{2}\right)}
$$

into Eq. (A1),

$$
\left|A_{i}\right|=\sqrt{\left(\omega_{s} / \omega_{i}\right)\left(\left|A_{s}\right|^{2}-\left|A_{s 0}\right|^{2}\right)}
$$

and

$$
\left|A_{p}\right|=\sqrt{\left|A_{p 0}\right|^{2}+\left(\omega_{s} / \omega_{p}\right)\left(\left|A_{s 0}\right|^{2}-\left|A_{s}\right|^{2}\right)}
$$

into Eq. (A2) and

$$
\left|A_{s}\right|=\sqrt{\left|A_{s 0}\right|^{2}+\left(\omega_{i} / \omega_{s}\right)\left|A_{i}\right|^{2}}
$$

and

$$
\left|A_{p}\right|=\sqrt{\left|A_{p 0}\right|^{2}-\left(\omega_{i} / \omega_{p}\right)\left|A_{i}\right|^{2}}
$$

into Eq. (A3) we obtain a set of three uncoupled equations which are solved by separation of the variables,

$$
\begin{aligned}
& \frac{d\left|A_{p}\right|}{\sqrt{\frac{\omega_{p}}{\omega_{i}}\left(\left|A_{p 0}\right|^{2}-\left|A_{p}\right|^{2}\right)\left[\left|A_{s 0}\right|^{2}+\frac{\omega_{p}}{\omega_{s}}\left(\left|A_{p 0}\right|^{2}-\left|A_{p}\right|^{2}\right)\right]}} \\
& =-\frac{\beta}{2} \frac{\omega_{s} \omega_{i}}{\omega_{0}^{2}} d x, \quad \text { (A17) } \\
& \frac{d\left|A_{s}\right|}{\sqrt{\frac{\omega_{s}}{\omega_{i}}\left(\left|A_{s}\right|^{2}-\left|A_{s 0}\right|^{2}\right)\left[\left|A_{p 0}\right|^{2}+\frac{\omega_{s}}{\omega_{p}}\left(\left|A_{s 0}\right|^{2}-\left|A_{s}\right|^{2}\right)\right]}} \\
& =\frac{\beta}{2} \frac{\omega_{i} \omega_{p}}{\omega_{0}^{2}} d x, \\
& \sqrt{\left[\left|A_{s 0}\right|^{2}+\frac{\omega_{i}}{\omega_{s}}\left|A_{i}\right|^{2}\right]\left[\left|A_{p 0}\right|^{2}-\frac{\omega_{i}}{\omega_{p}}\left|A_{i}\right|^{2}\right]} \\
& =\frac{\beta}{2} \frac{\omega_{s} \omega_{p}}{\omega_{0}^{2}} d x .
\end{aligned}
$$

The solutions (see, for example, Ref. [29]) can be expressed in terms of the Jacobi elliptic functions $\operatorname{sn}(u, \mathrm{k})$, $\mathrm{cn}(u, \mathrm{k})$, and $\operatorname{dn}(u, \mathrm{k})$, i.e.

$$
\begin{aligned}
\left|A_{p}\right| & =\left|A_{p 0}\right| \operatorname{sn}(K-g N / \mathrm{k}, \mathrm{k}), \\
\left|A_{s}\right| & =\sqrt{\frac{\omega_{p}}{\omega_{s}}}\left|A_{p 0}\right| \operatorname{dn}(K-g N / \mathrm{k}, \mathrm{k}) \\
& =\left|A_{s 0}\right| / \operatorname{dn}(g N / \mathrm{k}, \mathrm{k}), \\
\left|A_{i}\right| & =\sqrt{\frac{\omega_{p}}{\omega_{i}}}\left|A_{p 0}\right| \operatorname{cn}(K-g N / \mathrm{k}, \mathrm{k}) .
\end{aligned}
$$


Here $K$ is the complete elliptic integral of the first kind with the modulus value $\mathrm{k}$ given by Eq. (31). The complementary modulus value is

$$
\mathrm{k}^{\prime}=\sqrt{1-\mathrm{k}^{2}}=\sqrt{\omega_{s} A_{s 0}^{2} /\left(\omega_{s} A_{s 0}^{2}+\omega_{p} A_{p 0}^{2}\right)}
$$

and the ratio

$$
\mathrm{k}^{\prime} / \mathrm{k}=\sqrt{\omega_{s} / \omega_{p}} A_{s 0} / A_{p 0}
$$

while $g N$ stands for $\frac{\omega_{p} \sqrt{\omega_{s} \omega_{i}}}{\omega_{0}^{2}} \beta A_{p 0} N=\sqrt{1-\delta^{2}} g_{0} N$ (see Eqs. (17) and (20) in the main text). Formula Eq. (30) for power gain $G_{s}^{(\mathrm{d})}$ immediately follows from Eq. (A21).

The transmission line length ensuring maximum pos- sible power gain

$$
G_{s}^{\max }=\frac{1}{\mathrm{k}^{\prime 2}}=\frac{\omega_{s} A_{s 0}^{2}+\omega_{p} A_{p 0}^{2}}{\omega_{s} A_{s 0}^{2}}
$$

should correspond to the half of the period of the Jacobi elliptic function $\operatorname{dn}(u, \mathrm{k})=\operatorname{dn}(u+2 K, \mathrm{k})$ [30], i.e. $K$, so

$$
N_{m}=\frac{\mathrm{k}}{g} K(\mathrm{k}) \approx \frac{1}{g} \ln \frac{4}{\mathrm{k}^{\prime}} .
$$

For a longer transmission line, in our case at $N>N_{m}=$ 399 , the reverse power conversion from the signal and the idler to the pump occurs as shown in Fig. 3 (see also Fig. 6 in Ref. [19]).
[1] B. Yurke, L. R. Corruccini, P. G. Kaminsky, L. W. Rupp, A. D. Smith, A. H. Silver, R. W. Simon, and E. A. Whittaker, Observation of parametric amplification and deamplification in a Josephson parametric amplifier, Phys. Rev. A 39, 2519 (1989).

[2] R. Movshovich, B. Yurke, P. G. Kaminsky, A. D. Smith, A. H. Silver, R. W. Simon, and M. V. Schneider, Observation of zero-point noise squeezing via a Josephsonparametric amplifier, Phys. Rev. Lett. 65, 1419 (1990).

[3] M. A. Castellanos-Beltran and K. W. Lehnert, Widely tunable parametric amplifier based on a superconducting quantum interference device array resonator, Appl. Phys. Lett. 91, 083509 (2007).

[4] J. Mutus, T. White, R. Barends, Y. Chen, Z. Chen, B. Chiaro, A. Dunsworth, E. Jeffrey, and J. Kelly, Strong environmental coupling in a Josephson parametric amplifier, Appl. Phys. Lett. 104, 263513 (2014).

[5] M. Hatridge, R. Vijay, D. H. Slichter, John Clarke, and I. Siddiqi, Dispersive magnetometry with a quantum limited SQUID parametric amplifier, Phys. Rev. B 83, 134501 (2011).

[6] J. D. Teufel, T. Donner, Dale Li, J. W. Harlow, M. S. Allman, K. Cicak, A. J. Sirois, J. D. Whittaker, K. W. Lehnert and R. W. Simmonds, Sideband cooling of micromechanical motion to the quantum ground state, Nature 475, 359 (2011).

[7] B. Abdo, F. Schackert, M. Hatridge, C. Rigetti, and M. Devoret, Josephson amplifier for qubit readout, Appl. Phys. Lett. 99, 162506 (2011).

[8] C. Eichler, D. Bozyigit, and A. Wallraff, Characterizing quantum microwave radiation and its entanglement with superconducting qubits using linear detectors, Phys. Rev. A 86, 032106 (2012).

[9] B. Abdo, A. Kamal, and M. Devoret, Nondegenerate, three-wave mixing with the Josephson ring modulator, Phys. Rev. B 87, 014508 (2013).

[10] P. Krantz, A. Bengtsson, M. Simoen, S. Gustavsson, V. Shumeiko, W. D. Oliver, C. M. Wilson, P. Delsing, J. Bylander, Single-shot read-out of a superconducting qubit using a Josephson parametric oscillator, Nature Comm. 7, 11417 (2016).

[11] B. H. Eom, P. K. Day, H. G. LeDuc, and J. Zmuidzinas, A wideband, low-noise superconducting amplifier with high dynamic range, Nat. Phys. 8, 623 (2012).

[12] O. Yaakobi, L. Friedland, C. Macklin, and I. Siddiqi, Parametric amplification in Josephson junction embedded transmission lines, Phys. Rev. B 87, 144301 (2013).

[13] K. O'Brien, C. Macklin, I. Siddiqi, and X. Zhang, Resonant phase matching of Josephson junction traveling wave parametric amplifiers, Phys. Rev. Lett. 113, 157001 (2014).

[14] T. C. White, J. Y. Mutus, I.-C. Hoi, R. Barends, B. Campbell, Yu Chen, Z. Chen, B. Chiaro, A. Dunsworth, E. Jeffrey, J. Kelly, A. Megrant, C. Neill, P. J. J. O'Malley, P. Roushan, D. Sank, A. Vainsencher, J. Wenner, S. Chaudhuri, J. Gao and J. M. Martinis, Traveling wave parametric amplifier with Josephson junctions using minimal resonator phase matching, Appl. Phys. Lett. 106, 242601 (2015).

[15] M.T. Bell and A. Samolov, Traveling-wave parametric amplifier based on a chain of coupled asymmetric SQUIDs, Phys. Rev. Applied 4, 024014 (2015).

[16] C. Macklin, K. O'Brien, D. Hover, M. E. Schwartz, V. Bolkhovsky, X. Zhang, W. D. Oliver, I. Siddiqi, A nearquantum-limited Josephson traveling-wave parametric amplifier, Science 350, 307 (2015).

[17] E. Jeffrey, D. Sank, J. Y. Mutus, T. C. White, J. Kelly, R. Barends, Y. Chen, Z. Chen, B. Chiaro, A. Dunsworth, A. Megrant, P. J. J. O'Malley, C. Neill, P. Roushan, A. Vainsencher, J. Wenner, A. N. Cleland, and J. M. Martinis, Fast accurate state measurement with superconducting qubits, Phys. Rev. Lett. 112, 190504 (2014).

[18] G. P. Agrawal, Nonlinear fiber optics (Academic press, San Diego, California, 2007).

[19] A. L. Cullen, Theory of the travelling-wave parametric amplifier, Proc. IEE - Part B: Electron. and Communication Eng. 107, 101 (1960).

[20] M. R. Vissers, R. P. Erickson, H.-S. Ku, L. Vale, Xian Wu, G. C. Hilton, and D. P. Pappas, Low-noise kinetic inductance traveling-wave amplifier using three-wave mixing, Appl. Phys. Lett. 108, 012601 (2016).

[21] The SQUID handbook Vol.1: Fundamentals and technology of SQUIDs and SQUID systems, J. Clarke, A. I. Braginski eds. (Weinheim, WILEY-VCH, 2004).

[22] K.K. Likharev, Dynamics of Josephson junctions and circuits (Gordon and Breach, New York, 1986). 
[23] P. A. Franken, A. E. Hill, C. W. Peters, and G. Weinreich, Generation of optical harmonics, Phys. Rev. Lett. 7, 118 (1961).

[24] D. N. Klyshko, Coherent photon decay in a nonlinear medium, JETP Lett. 6, 23 (1967).

[25] D. C. Burnham and D. L. Weinberg, Observation of simultaneity in parametric production of optical photon pairs, Phys. Rev. Lett. 25, 84 (1970).

[26] D. N. Klyshko, Photons and nonlinear optics (Gordon and Breach, New York, 1988).

[27] R. Klopfenstein, A transmission line taper of improved design, Proc. IRE 44, 31 (1956).

[28] J. M. Manley and H. E. Rowe, Some general properties of nonlinear elements, part 1. General energy relations, Proc. IRE 44, 904 (1956).

[29] O. V. Rudenko and S. I. Soluian, Theoretical foundations of nonlinear acoustics (Springer US, New York, 1977).

[30] I. S. Gradshteyn and I. M. Ryzhik, Table of integrals, series, and products, edited by A. Jeffrey and D. Zwillinger (Academic Press, New York, 2007). 